\title{
Estudo de Definição Alternativa da Probabilidade de Permanência no Rebanho para a Raça Nelore ${ }^{1}$
}

\author{
Cintia Righetti Marcondes²,3, João Cláudio do Carmo Paneto 2,3, Luiz Antônio Framartino Bezerra 2,4, \\ Raysildo Barbosa Lôbo ${ }^{2}$
}

\begin{abstract}
RESUMO - Uma definição alternativa de permanência no rebanho foi estudada em comparação à definição usual. Na definição usual, foram consideradas vacas com pelo menos três partos até os 76 meses de idade como valor 1 e vacas com menos de 3 partos com valor zero. A definição alternativa considerou os valores 0, 1, 2 e 3, respectivamente, para vacas com menos de três, com três, quatro ou cinco partos até os 76 meses de idade. Foram obtidas estimativas de herdabilidade e preditas as DEPs (Diferenças Esperadas na Progênie) para probabilidade de permanência no rebanho de 4.180 touros com filhas na base de dados do Programa de Melhoramento Genético da Raça Nelore (PMGRN-USP). Utilizaram-se o modelo linear unicaráter de touro avô-materno e a análise Bayesiana, por meio do software MTGSAM_threshold (Multiple-Trait Gibbs Sampler for Animal Models). Considerou-se tamanho de cadeia de Gibbs de 225 mil, período de descarte amostral de 25 mil e tomada de amostra a cada 1.000 rodadas. A correlação entre classificações foi calculada por meio do SAS. As estimativas de herdabilidade foram de menor magnitude para a característica padrão (0,07 vs 0,08). A correlação de posto foi de $87,5 \%$, ou seja, houve considerável alteração na classificação dos touros avaliados. Dados os valores das estimativas de variância de touro, de herdabilidade e de variações nas DEPs preditas, concluiu-se que a característica alternativa de permanência no rebanho possui maior capacidade de detecção da variabilidade genética que a característica padrão, sob modelo linear.
\end{abstract}

Palavras-chave: bayesiana, bovinos, modelo linear, Nelore, probabilidade de permanência no rebanho

\section{Alternative Definition of Stayability for Nelore Beef Cattle}

ABSTRACT - An alternative definition of stayability was compared to an usual one. The usual definition in this study classifies the cows with at least three calvings at age of 76 months as success, setting them a value 1 , and the ones with less than three calvings at this age as failure, setting them a value 0 . The alternative definition sets the cows the values $0,1,2$, or 3 , according to the number of calvings: less than three, three, four, or five calves at age of 76 months, respectively. Heritability estimates and EPDs (Expected Progeny Differences) of stayability were obtained using the information of 4,180 bulls with daughters in the database of the genetic breeding program of the Nellore cattle (PMGRN-USP). Both maternal grandsire single trait linear model and bayesian analysis were part of the MTGSAM_threshold (Multiple-Trait Gibbs Sampler for Animal Models) software. The implementation considered a length of Gibbs chain of 225 thousand, a period of burn-in of 25 thousand and a thinning interval at each 1 thousand runs. The correlation between both ranks was calculated by SAS (Statistical Analysis System). The heritability estimates were 0.07 to the usual definition of the trait versus 0.08 to the alternative one. The rank correlation was $87.5 \%$, with considerable change on bull classifications. According to the estimates of sire variance and heritability and variations on EPDs, it can be concluded that the alternative definition of stayability has greater capacity to detect genetic variation under a linear model.

Key Words: bayesian, beef cattle, linear model, Nelore, stayability

\section{Introdução}

Para que o empreendimento em gado de corte apresente lucratividade, a vaca deve permanecer em produção até que seus custos de recria e de manutenção sejam pagos (Ritchie, 1995; Snelling et al., 1995; Formigoni et al., 2002; Mwansa et al., 2002). Nos programas de melhoramento atuais, maiores exigências são colocadas nos critérios de seleção de fêmeas.
Um destes critérios é a stayability, ou probabilidade de permanência no rebanho, ou ainda longevidade produtiva, vida útil no rebanho ou qualquer uma das várias denominações que caracterizem resumir, em rebanhos leiteiros ou de corte, a vida produtiva de uma fêmea no rebanho. Poucos são os estudos sobre permanência no rebanho para a raça Nelore e mais raros são aqueles sob enfoque Bayesiano na área de produção animal. Com o desenvolvimento de compu-

\footnotetext{
${ }_{1}^{1}$ Parte da tese de Doutorado da primeira autora.

2 Dep. de Genética da FMRP-USP. E.mail: cimarcon@yahoo.com

3 Zootecnistas.

${ }^{4}$ Analista de Sistemas.
} 
tadores mais rápidos e com maior capacidade de memória, além de softwares de livre-acesso, esta área, pouco explorada pelos pesquisadores em melhoramento animal, tem se tornado muito atraente, por permitir investigações desde a melhor definição para a característica até o cálculo de ganho genético.

A importância econômica da permanência no rebanho para a seleção de vacas Nelore está descrita na literatura (Formigoni et al., 2002; Paneto et al., 2002). O seu uso como critério de seleção, considerando-se os valores das estimativas de herdabilidade (Silva et al., 2003a,b; Marcondes, 2003; Marcondes et al., 2005), pode ser recomendado, especialmente por meio da predição de DEPs dos touros, apesar do possível incremento no intervalo de gerações, se utilizada para seleção direta.

A definição usual da permanência no rebanho, por tratar-se de característica binária (neste estudo, assumindo valor 1 para vacas com pelo menos três partos até os seis anos de idade e valor 0 para as que não atingiram esta meta), exige maior demanda de tempo para as análises e a aplicação de metodologias específicas, como a desenvolvida por Van Tassel et al. (1998). Além disso, a diferenciação entre vacas codificadas com valor 1 , mas que podem ter parido três, quatro ou cinco vezes, tendo-se em vista a grande variabilidade genética existente na raça quanto aos aspectos reprodutivos, torna-se o foco de estudo deste trabalho. Diferentes valores atribuídos possibilitam avaliar as diferenças de variabilidade genética na característica de permanência no rebanho ou ainda, as diferenças na classificação dos touros, quando comparados à definição usual ou padrão (0 ou 1).

\section{Material e Métodos}

O arquivo de dados continha 54.727 registros de produção de vacas nascidas entre 1986 e 1996 em rebanhos participantes do Programa de Melhoramento Genético da Raça Nelore (PMGRN-USP; Lôbo et al., 2002). O grupo de contemporâneos ao nascimento foi composto por Fazenda e Ano de nascimento da vaca. A consistência dos dados, realizada por meio do programa Statistical Analysis System (SAS, 2000), considerou touros com pelo menos duas filhas dentro do mesmo grupo de contemporâneos (GC) e grupos com pelo menos dois touros diferentes. Além disso, foram eliminados grupos com médias 0 ou 1 para permanência no rebanho, isto é, sem variabilidade dentro do grupo de contemporâneos.
A característica probabilidade de permanência no rebanho padrão foi codificada com valor 1 para vacas com pelo menos três partos até os 76 meses de idade ou valor zero para as que tiveram menos de três partos. Segundo Formigoni et al. (2002), com três partos, os custos de cria e recria da vaca estariam pagos. Os 76 meses foram considerados para que as vacas alcançassem três partos, uma vez que, em média, o primeiro parto ocorre aos 32 meses de idade.

Para a característica alternativa de probabilidade de permanência no rebanho, foram atribuídos os valores $0,1,2$ e 3 para vacas, respectivamente, com menos de três partos e com três, quatro ou cinco partos até os 76 meses de idade.

Os arquivos foram analisados por meio do MTGSAM for threshold (Multiple-Trait Gibbs Sampler for Animal Models, Van Tassell et al., 1998), sob modelo linear unicaráter de touro-avô materno, para obtenção de componentes de (co)variância, estimativas de herdabilidade e soluções para cada touro (que originam as Diferenças Esperadas na Progênie - DEPs -, como médias posteriores). A implementação adequada à base de dados do PMGRN-USP foi realizada considerando-se tamanho de cadeia de Gibbs de 225 mil, período de descarte amostral de 25 mil e tomada de amostra a cada 1.000 rodadas.

As análises dos dados das amostras das distribuições posteriores foram realizadas por meio do programa GIBANAL, desenvolvido por Van Kaam (1997).

As descrições das características padrão e alternativa encontram-se nas Tabelas 1 e 2.

Por tratar-se de análise unicaráter, as relações entre as variâncias estimadas sob modelo touro-avô materno foram a variância genética aditiva, considerada como $4 \sigma^{2} s$ (variância de touro), e a variância

Tabela 1 - Números de registros (REG), de grupos de contemporâneos (GC), de touros e de avós maternos, para as características de permanência padrão e alternativa

Table 1 - Number of observations (Obs), number of contemporary groups (CG) and number of sires and maternal-grandsires (MGS) in files to standard and alternative stayabilities

\begin{tabular}{lcccc}
\hline $\begin{array}{l}\text { Característica } \\
\text { Trait }\end{array}$ & REG & GC & TOUROS & AVÔS \\
Padrão & CG & Sires & MGS \\
\hline Alternativa & 28959 & 678 & 1682 & 480 \\
& 28994 & 683 & 1683 & 480 \\
\hline
\end{tabular}


Tabela 2 - Números de registros (REG) e freqüência (em \%) dos diferentes valores atribuídos à característica de permanência alternativa

Table 2 - Number of observations (Obs) and frequency (in \%) for different values in alternative stayability

\begin{tabular}{lcc}
\hline $\begin{array}{l}\text { Valor } \\
\text { Value }\end{array}$ & REG & $\begin{array}{c}\text { Freqüência (\%) } \\
\text { Frequency }\end{array}$ \\
\hline 0 & 18332 & 63,23 \\
1 & 8256 & 28,48 \\
2 & 2357 & 08,13 \\
3 & 49 & 00,16 \\
Total & 28994 & 100 \\
\hline
\end{tabular}

fenotípica, como a soma de $\left(\sigma^{2} s+1 / 4 \sigma^{2} s\right)$ à variância residual $\left(\sigma^{2} e\right)$, e, conseqüentemente, a herdabilidade, estimada sob modelo touro como a fração herdável da variância total existente.

Correlações entre classificações para as DEPs (correlação de posto ou correlação de Spearman) foram obtidas pelo procedimento CORR, opção Spearman do SAS ${ }^{\circledR}$.

\section{Resultados e Discussão}

Constam na Tabela 3 as estimativas dos componentes genéticos (variância de touro e residual) e da herdabilidade, sob modelo linear, para as duas definições de permanência no rebanho: padrão (0 ou 1) e alternativa $(0,1,2$ ou 3 , conforme o número de partos).

Os valores médios, as modas, medianas, amplitudes (valores mínimos e máximos) eas regiões de credibilidade a 95\%, em relação às variâncias de touro, residual e à herdabilidade, foram maiores para a característica alternativa. Observa-se, por exemplo, que os valores da variância de touro obtidos para a característica alternativa são o dobro daqueles para a característica padrão e, ainda neste caso, a região de credibilidade a 95\% não é coincidente.

Os gráficos das distribuições posteriores das herdabilidades (Figura 1) mostram que a característica alternativa (b) de permanência apresentou duas modas bem próximas (de 0,07 a 0,08), o mesmo não ocorrendo para a característica padrão (a).

Tabela 3 - Descrição dos dados e das estimativas dos componentes genéticos (variância de touro, $\sigma^{2}$ s, e variância residual, $\left.\sigma^{2} \mathrm{e}\right)$ e herdabilidades $\left(\mathrm{h}^{2}\right)$, obtidas na análise Bayesiana da permanência no rebanho, sob modelo linear, para ambas as definições

Table 3 - Data description and (co)variance components (sire, $\sigma^{2} s$, and residual variances, $\sigma^{2} e$ ) and heritabilities $\left(h^{2}\right)$, in Bayesian analysis for stayability, under linear model for two different definitions

\begin{tabular}{|c|c|c|c|}
\hline & & $\begin{array}{l}\text { Padrão } \\
\text { Standard }\end{array}$ & $\begin{array}{l}\text { Alternativa } \\
\text { Alternative }\end{array}$ \\
\hline \multirow[t]{8}{*}{$\sigma^{2} s$} & $\begin{array}{l}\text { Descrição dos dados (média } \pm \text { desvio-padrão) } \\
\text { Data description (mean }+ \text { s.d.) }\end{array}$ & $0,37 \pm 0,48$ & $0,45 \pm 0,65$ \\
\hline & Correlação serial(serial correlation) & 0,055 & $-0,047$ \\
\hline & Média (Mean) & 0,003 & 0,007 \\
\hline & Moda (Mode) & 0,003 & 0,006 \\
\hline & Mediana (Median) & 0,003 & 0,007 \\
\hline & Mínimo (Minimum) & 0,002 & 0,004 \\
\hline & Máximo (Maximum) & 0,005 & 0,011 \\
\hline & Região de credibilidade a 95\% (Credible region at 95\%) & 0,002 a 0,005 & 0,005 a 0,010 \\
\hline \multirow[t]{8}{*}{$\sigma^{2} e$} & Correlação serial (serial correlation) & $-0,098$ & $-0,062$ \\
\hline & Média (Mean) & 0,191 & 0,339 \\
\hline & Moda (Mode) & 0,190 & 0,339 \\
\hline & Mediana (Median) & 0,191 & 0,338 \\
\hline & Mínimo (Minimum) & 0,187 & 0,331 \\
\hline & Máximo (Maximum) & 0,196 & 0,347 \\
\hline & Região de credibilidade a 95\% (Credible region at 95\%) & 0,188 a 0,194 & 0,333 a 0,345 \\
\hline & Correlação serial(serial correlation) & 0,056 & $-0,049$ \\
\hline \multirow{6}{*}{$h^{2}$} & Média (Mean) & 0,066 & 0,079 \\
\hline & Moda (Mode) & & \\
\hline & Mediana (Median) & 0,065 & 0,078 \\
\hline & Mínimo (Minimum) & 0,035 & 0,051 \\
\hline & Máximo (Maximum) & 0,108 & 0,126 \\
\hline & Região de credibilidade a 95\% (Credible region at 95\%) & 0,042 а 0,094 & 0,055 a 0,118 \\
\hline
\end{tabular}


(a)

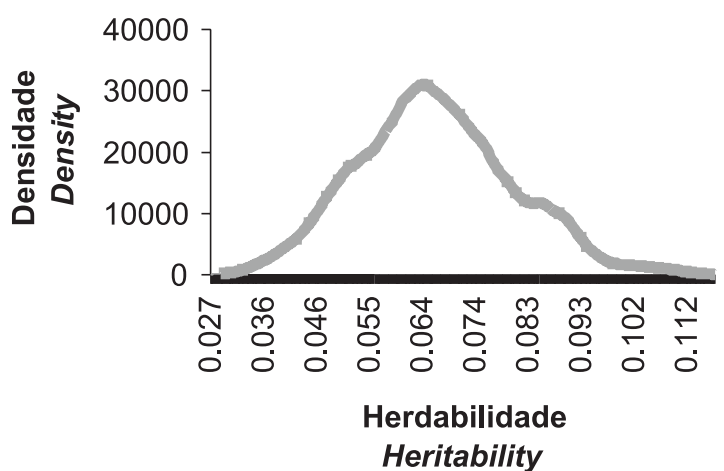

(b)

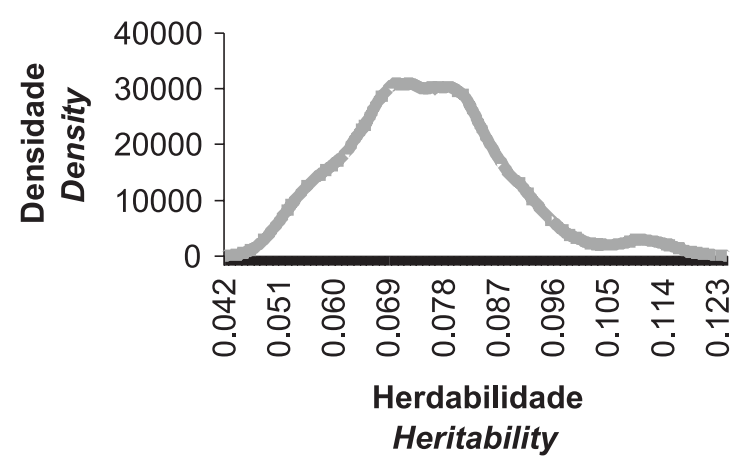

Figura 1 - Distribuições posteriores da herdabilidade para características de permanência padrão (a) e alternativa (b), sob modelo linear.

Figure 1 - Marginal posterior distribution for standard (a) and alternative (b) stayability, under linear model.

Comparando-se as estimativas de herdabilidade, nota-se que os valores mínimos e máximos foram superiores para a característica alternativa $(0,051$ vs 0,035, no valor mínimo, e 0,126 vs 0,108 , no valor máximo da herdabilidade), resultado possivelmente da melhor detecção de variabilidade de permanência alternativa no rebanho.

Na Tabela 4 encontra-se a descrição das DEPs como desvios em relação à base genética.

A correlação de posto foi igual a $84,5 \%$, ou seja, houve considerável alteração na classificação dos 4.180 touros avaliados. Quando a mesma correlação foi aplicada sobre $1 \%$ dos melhores (42 touros, TOP $1 \%$ ) e sobre $1 \%$ dos piores touros, os valores ficaram acima de 99,9\%. A característica alternativa mostrou-se mais eficiente na detecção da variabilidade existente entre os touros avaliados, por disponibilizar valores genéticos com maior amplitude em sua distribuição (variando de -0,1449 a 0,1580), ou seja, o pior touro seria $14 \%$ inferior à média e o melhor, $16 \%$ superior à média dos touros avaliados.

Tabela 4 - Estatística descritiva das DEPs (Diferenças Esperadas na Progênie, em porcentagem) dos touros ( $\mathrm{N}=4.180)$, obtidas para ambas as definições

Table 4 - Descriptive analysis for DEPs to sires $(N=4,180)$, for two different definitions

\begin{tabular}{lcc}
\hline $\begin{array}{l}\text { Estatística } \\
\text { Parameter }\end{array}$ & $\begin{array}{c}\text { Padrão } \\
\text { Standard }\end{array}$ & $\begin{array}{c}\text { Alternativa } \\
\text { Alternative }\end{array}$ \\
\hline $\begin{array}{l}\text { Média } \\
\text { Mean }\end{array}$ & 0,0018 & 0 \\
$\begin{array}{l}\text { Desvio-padrão } \\
\text { Standard deviation }\end{array}$ & 0,0159 & 0,0243 \\
$\begin{array}{l}\text { Mínimo } \\
\text { Minimum }\end{array}$ & $-0,099$ & $-0,1449$ \\
$\begin{array}{l}\text { Máximo } \\
\text { Maximum }\end{array}$ & 0,1017 & 0,1580 \\
\hline
\end{tabular}

\section{Conclusões}

As comparações sob modelo linear entre as características padrão e a alternativa (originada da percepção de diferenças em fertilidade entre as vacas codificadas com valor 1) comprovaram que alterações na classificação de um número significativo de touros avaliados podem ocorrer, mesmo que nas posições intermediárias do posto, talvez pela maior eficiência da análise da característica alternativa em detectar maior variabilidade entre touros.

\section{Agradecimento}

Ao PRONEX, à FAPESP, ao CNPq, à FINEP e à ANCP, pelo apoio financeiro, e aos criadores do PMGRN.

\section{Literatura Citada}

FORMIGONI, I.B.; SILVA, J.A. II V.; BRUMATTI, R.C. et al. Economic aspects of stayability as selection criterion in beef cattle industry in Brazil. In: WORLD CONGRESS ON GENETICS APPLIED TO LIVESTOCK PRODUCTION, 7., 2002, Montpellier - França. Anais... Montpellier: 2002. CD-ROM. Seção 2, Comunicação 02-62.

MARCONDES, C.R. Análise Bayesiana da probabilidade de permanência no rebanho como característica de seleção para a raça Nelore. Ribeirão Preto: Universidade de São Paulo, 2003. 100p. Tese (Doutorado em Ciências Biológicas, Genética) - Universidade de São Paulo, 2003.

MARCONDES, C.R.; PANETO, J.C.C.; OLIVEIRA, H.N. et al. Análise Bayesiana da stayability na raça Nelore: implementações para tamanho de cadeia, período de descarte e tomada de amostra. In: CONGRESSO NACIONAL DE GENÉTICA, 49., 2003, Águas de Lindóia. Anais... Ribeirão Preto: SBG, 2003a. CD-ROM. Genética Animal. GA-260. 
MARCONDES, C.R.; PANETO, J.C.C.; SILVA, J.A. II V. et al. Comparação entre análises para stayability de vacas Nelore como modelo linear e como modelo de limiar. Arquivo Brasileiro de Medicina Veterinária e Zootecnia, v.57, n.2, p.234-240, 2005.

MWANSA, P.B.; CREWS JR., D.H.; WILTON, J.W. et al. Multiple trait selection for maternal productivity in beef cattle. Jounal of Animal Breeding and Genetics, v.119, p.391-399, 2002.

PANETO, J.C.C.; SILVA, J.A. II V.; BEZERRA, L.A.F. et al. Expected response to selection on stayability and its economic weight in a population of Nelore cattle in Brazil. In: WORLD CONGRESS ON GENETICS APPLIED TO LIVESTOCK PRODUCTION, 7., 2002, Montpellier. Anais... Montpellier: França, 2002. CD-ROM. Seção 2, Comunicação 02-67.

RITCHIE, H. The search for the elusive optimum cow. Angus Journal, USA, p.143-145, outubro 1995. Disponível em: <http://www.angusjournal.com/ArticlePDF/ 1095 OptimumCow.pdf $>$. Acesso em: 07 nov. 2003.

SILVA, J. A. II V.; ELER, J.P.; FERRAZ, J.B.S. et al. Heritability estimate for stayability in Nelore cows. Livestock Production and Science, v.79, n.1, p.97-101, 2003a.
SILVA, J.A. II V.; ELER, J.P.; OLIVEIRA, H.N. et al. Análise genética da habilidade de permanência em fêmeas da raça Nelore. Revista Brasileira de Zootecnia, v.32, n.3, p.598-604, 2003b.

SNELLING, W.M.; GOLDEN, B.L.; BOURDON, R.M. Withinherd genetic analysis of stayability of beef females. Journal of Animal Science, v.73, p.993-1001, 1995.

Van KAAM, J.B.C.H.M. GIBANAL: Analyzing program for Markov Chain Monte Carlo sequences version 2.3, 1997.

Van TASSELL, C.P.; Van VLECK, L.D.; GREGORY, K.E. Bayesian analysis of twinning and ovulation rates using a multiple-trait threshold model and Gibbs sampling. Journal of Animal Science, v.76, p.2048-2061, 1998.

Recebido em: 18/02/04

Aceito em: 20/04/05 